O9 (continued)

for-profits, universities, and hospitals, partner with schools to provide nutrition education programs (NEPs).

Objective: This study assessed NEPs in NYC schools. Study Design, Setting, Participants, Intervention: Cross sectional study of the 40 organizations that run 101 NEPs in NYC schools in the 1,840 NYC public schools.

Outcome Measures and Analysis: Survey completed by organizations that run NEPs with questions on organizational structure, program characteristics, and schools worked with during 2016-17 school year; and publically available data from NYC Department of Education on school demographics. Descriptive statistical analysis.

Results: Most NEPs surveyed (68\%) have $\leq 10$ full time staff. Forty-three percent of NEPs started after 2011. Common student activities include cooking (69\%), classroom lessons (66\%), gardening (46\%), and field trips (31\%). Reported barriers include limited time for NEPs (37\%) and limited space for implementation (34\%). Overall, 56\% of NYC schools had at least one NEP. Only $27 \%$ of schools had more than one. NEPs were evenly distributed across schools with varying percentage of students qualifying for free/reduced price lunch and percentage Black and Hispanic students. NEPs varied by school type: $69 \%$ of elementary schools had one or more NEP; $50 \%$ of middle schools; and $32 \%$ of high schools. A previous study from 2011-2012 found 39\% of elementary schools in the NYC boroughs of Brooklyn, Manhattan and Queens had one or more NEPs. An analysis of elementary schools in these boroughs for the 2016-2017 data found a rate of $71 \%$.

Conclusions and Implications: There are not enough NEPs being implemented in NYC schools for students to receive the full benefits of nutrition education. Policy makers, researchers, funders, and advocates need to effectively collaborate for more schools to have more NEPs. This study can be a model for other cities, regions, or states to conduct landscape assessments of NEPs.

Funding: New York State Health Foundation.

\section{Food Waste in Schools: An Intervention of Randomized Schools on the Reduction of Food Waste}

Sara Elnakib, MPH, RD, CHES, elnakib@njaes.rutgers.edu, Rutgers Cooperative Extension, 1310 Route 23 North, Wayne, NJ 07470; Mariel Mendez, MPH

Background (Background, Rationale, Prior Research, and/or Theory): The USDA estimates that 30 to $40 \%$ of the food produced in the United States is wasted, resulting in a loss of $\$ 218$ billion dollars in food. In addition to the economic cost of food, there is an additional cost in resources such as land, water, labor and transport that is not often factored into the cost of food waste. Foodwaste is also the leading emitter of methane in the world. Methane is a greenhouse gas more potent than carbon dioxide.
Objective: This study sought to address many of the gaps in the current research on food waste intervention programs in schools by measuring a large sample size, measuring each meal component and randomizing the selection of schools participating.

Study Design, Setting, Participants, Intervention: The school district used for this intervention has a total of 46 schools of which 30 schools were elementary/ middle schools that prepare their meals on-site. A randomly selected 15 of the 30 eligible schools participated in the study. Data collection occurred one month prior to the intervention training from February 16, 2017 until March 8,2017 . Afterwards we conducted a training session on March 10, 2017 for the food service workers and March 22, 2017 for the lunchroom monitors. Finally we measured the post intervention food waste from April 4, 2017 until April 28, 2017.

Outcome Measures and Analysis: A total of 9,140 trays were measured for foodwaste, 4,637 for the pre-intervention and 4,503 trays for the post-intervention.

Results: Of the food and beverages served during our 60 visits to schools 2,473 pounds were wasted before the intervention and 2,123 were wasted after the intervention. Overall, 350 pounds of food was saved which was a $14 \%$ reduction in food waste due to this intervention.

Conclusions and Implications: Overall the intervention showed the impact of a food service training program on reducing foodwaste. One of the limitations was that the study only measured plate/traywaste and did not include production waste.

Funding: None.

\section{Student Showcase}

\section{Hunger and Environmental Nutrition (HEN) Garden Lab: Participant Outcomes in a Garden Literacy Program Targeting Nutrition and Dietetics Students \\ Virginia Woollens, MS, RD, Appalachian State University; Maggie Gartman, MS, RDN; Lanae Hood, PhD, klball@meredith.edu, Meredith College, 3800 Hillsborough Street, Raleigh, NC 27607; Nadine Gregory, BS, Appalachian State University; Carla Toolan, BS; Kyle Hood, BS, Meredith College}

Objective: Although gardens are now common in K-12 farm-to-school initiatives, there currently is a deficit of research on initiatives targeting adult, college-aged students. The goal of this project was to identify which teaching practices best improved garden literacy and self-efficacy in nutrition and dietetics students.

Target Audience: Nutrition and dietetic students $(\mathrm{n}=30)$. Theory, Prior Research, Rationale: This study used a community-based participatory approach that guided development and implementation of the HEN Garden Lab education and teaching methods.

Continued on page S6 


\section{1 (continued)}

Description: The curriculum was revised and repeated over three consecutive years with three different cohorts of students. Teaching style differed each year, beginning with cooperative extension and by the third year, peer education. Participants completed a pre/post program questionnaire to collect information related to demographics, self-efficacy (0-10 scale), and garden literacy.

Evaluation: The sample was divided into three subgroups according to the program year. Data was analyzed using Microsoft Excel and interpreted by percent change calculations. The first year showed that pre/post program quiz scores showed an average percent increase in garden literacy by $21 \%$ and confidence in teaching sustainability concepts by 63\%. Confidence outcomes for teaching diverse populations (e.g. children, adult, elderly, and low-income) increased by $23.5 \%$ in the second year. Third-year results included an increase in garden literacy at 20\%, participant gardening self-efficacy at 139\%, and participant cooking confidence at $17 \%$.

Conclusions and Implications: Each year's teaching style and related curriculum resulted in different participant outcomes. The first and third year had the most impact on garden literacy changes but the second year resulted in the highest participant efficacy for teaching diverse populations. This program could guide the incorporation of garden-based education into undergraduate nutrition and dietetic internship programs while improving garden literacy and confidence in nutrition education among future nutrition professionals.

Funding: None.

\section{Innovative Approaches to the Evaluation of Hands-on Cooking Skills with Youth}

Jessica Metcalfe, MPH, jarick2@illinois.edu, University of Illinois, Urbana-Champaign, 904 W Nevada Street, MC081, Urbana, IL 61801; Barbara Fiese, PhD; Ruyu Liu, BS; Emilie Emberton, BS; Jennifer McCaffrey, PhD, MPH, RD

Objective: Evaluate the effectiveness of the Illinois Junior Chefs (IJC) program through pre- and post-intervention surveys and hands-on cooking skills assessments.

Target Audience: SNAP-Ed eligible youth $(n=419)$ aged 8-14.

Theory, Prior Research, Rationale: Experiential Learning Theory and Social Cognitive Theory suggest that educational experiences that allow for hands-on practice of skills such as cooking result in increased skill development. Past research with IJC and other culinary education programs shows that they can be effective in improving dietary attitudes and behaviors, but hands-on cooking skills themselves are rarely assessed, especially with youth populations. These types of assessments are crucial to determining whether participants in hands-on cooking programs actually improve their tangible culinary skills.

Description: IJC is a statewide culinary and nutrition education program that was implemented during summer 2017 by the University of Illinois Office of Extension and Outreach. Each of the five 2-hour IJC lessons has a food group focus (e.g. fruits), paired with an associated culinary skill (e.g. knife skills) and hands-on recipe preparation (e.g. fruit salsa). IJC was implemented in a variety of community settings, such as summer camps, Extension offices, and community centers.

Evaluation: Pre- and post-intervention surveys indicated that participants experienced significant improvements in cooking self-efficacy $(\mathrm{t}(417)=14.60, P<.001)$, cooking attitudes $(\mathrm{t}(411)=5.88, P<.001)$, fruit and vegetable preferences $(\mathrm{t}(402)=3.27, P=.001)$, self-efficacy for selecting and eating healthy foods $(\mathrm{t}(397)=5.12, P<.001)$, and cooking behaviors $(\mathrm{t}(392)=2.12, P=.035)$. Assessments of hands-on cooking skills also indicated that participants experienced increases in skills including measuring, cracking eggs, peeling, grating, and mixing techniques.

Conclusions and Implications: Participants in IJC experienced positive changes in cooking skills as well as dietary attitudes and behaviors. These findings will be used to build the evidence base for IJC and inform curriculum modifications in preparation for wider dissemination of the program.

Funding: Supplemental Nutrition Assistance Program-Education.

\section{Exploring Food Waste at a Residential Youth Summer Camp}

Susan Chen, BS, schen518@vt.edu, Virginia Tech, 121

Lucas Drive, Blacksburg, VA 24060; Elena Serrano, PhD

Background (Background, Rationale, Prior Research, and/or Theory): Up to $40 \%$ of all edible food is wasted in the U.S., mostly from consumer waste. Research on food waste is in its infancy, particularly among youth. Summer camps offer a unique setting for foodbased education with the opportunity to reach large numbers of youth.

Objective: The purpose of this study was to assess food waste in a youth camp setting; and determine if an educational program would reduce food waste.

Study Design, Setting, Participants, Intervention: The research team and camp staff developed and adapted experiential-based activities on food waste that aligned with the camp environment. This cross-sectional study included 864 residential campers, aged 9-13. Participants attended one of the four weekly sessions that were offered. All participants attended the same summer camp.

Outcome Measures and Analysis: Waste from three meals (breakfast, lunch, and dinner) was collected over a 24-hour cycle, before and after the program. Food waste was collected using the direct weighing method. Waste was separated into two categories, consumer and production waste.

Results: Although a decrease in consumer food waste was observed, results were not significant $(P=.06)$. Total consumer waste for all four weeks before and after the intervention was $76.5 \mathrm{lb}$ and $57.3 \mathrm{lb}$, respectively. Total production waste decreased from $441.5 \mathrm{lb}$ before to $390.6 \mathrm{lb}$ after the intervention. Production waste also decreased throughout the course of the four weeks.

Continued on page $S 7$ 